Nota Científica

\title{
Nuevos registros de distribución para Uranotaenia sapphirina (Osten Sacken, 1868) (Diptera: Culicidae) en Quintana Roo, México
}

\author{
New distributional records of Uranotaenia sapphirina (Osten Sacken, 1868) \\ (Diptera: Culicidae) in Quintana Roo, Mexico
}

\begin{abstract}
Karla Leticia Canto-Mis ${ }^{1}$, Rahuel J. Chan-Chable ${ }^{1^{*}}$ (D) , Ángel S. Gómez-Rivera ${ }^{1,2}$ (D) , Xinia Yazmín López-Sosa $^{3}$, Cassandra González-Acosta ${ }^{4}$, Fabián Correa-Morales ${ }^{4}$ y Pedro C. Mis Ávila ${ }^{1}$ (iD
\end{abstract}

${ }^{1}$ Servicios Estatales de Salud de Quintana Roo, Av. Héroes de Chapultepec 267, Centro, C.P. 77000, Chetumal, QuintanaRoo, México.E-mail:karla.miis94@gmail.com; 隄*rahuel_jere_1990@hotmail.com; asgomezrivera@ gmail.com; pedrochristianmis@gmail.com. Instituto Tecnológico de la Zona Maya, Tecnológico Nacional de México, Carretera Chetumal-Escárcega km 21.5, C.P. 77965, Ejido Juan Sarabia, Quintana Roo, México. ${ }^{3}$ Laboratorio Estatal de Salud Pública de Quintana Roo, Av. Chetumal S/N, Residencial, C.P. 77039, Chetumal, Quintana Roo, México. E-mail: xinia81@hotmail.com. ${ }^{4}$ Centro Nacional de Programas Preventivos y Control de Enfermedades, Eje 4 Sur, Av. Benjamín Franklin, Escandón I Secc, C.P. 06170, Ciudad de México, México. E-mail:cgonzalez_vectores@hotmail.com; fabiancorrea@msn.com

\section{ZooBank: urn:lsid:zoobank.org:pub:0D0014F9-D252-43F2-9044-799AC8844EDE https: / / doi.org/10.35249/ rche.47.3.21.19}

Resumen. Se realizaron colectas de larvas de culícidos en tres localidades del estado de Quintana Roo, México (Leona Vicario, Cacao y Ramonal) durante los meses de abril y mayo de 2021. Se identificó la especie Uranotaenia sapphirina, con lo cual se aportan nuevas localidades y se actualiza la lista de culícidos presentes en Quintana Roo a 87 especies.

Palabras clave: Distribución; mosquitos; Uranotaeniini.

Abstract. Culicidae larvae collections were carried out in three localities in the state of Quintana Roo, Mexico (Leona Vicario, Cacao and Ramonal) during the months of April and May 2021. The species Uranotaenia sapphirina, was identified, with this records new locations are provided and updating the list of Culicidae present in Quintana Roo to 87 species.

Key words: Distribution; mosquitoes; Uranotaeniini.

El género Uranotaenia Lynch Arribálzaga, 1891 pertenece a la tribu Uranotaeniini dentro de la subfamilia Culicinae (Knight y Stone 1977), incluye 271 especies descritas hasta el momento, agrupadas en los subgéneros Pseudoficalbia Theobald, 1912 y Uranotaenia Lynch Arribálzaga, 1891. El subgénero Pseudoficalbia agrupa 150 especies y Uranotaenia a 121 especies (Harbach 2021).

Los estados inmaduros del género Uranotaenia generalmente se desarrollan en diferentes hábitats acuáticos como pantanos, cuerpos de agua temporales con vegetación, agujeros de rocas, agujeros de árboles y contenedores artificiales (Hoshi et al. 2014). Los adultos reposan cerca de los hábitats larvales, sobre superficies húmedas poco expuestas, y pueden

Recibido 13 Agosto 2021 / Aceptado 8 Septiembre 2021 / Publicado online 30 Septiembre 2021 Editor Responsable: José Mondaca E. 
encontrarse en grandes cantidades si los refugios son adecuados. Aunque se desconocen las preferencias alimenticias de la mayoría de las especies de Uranotaenia, se han señalado como hospedantes a reptiles, anfibios, aves y mamíferos (Burkett-Cadena 2013).

La distribución del género Uranotaenia incluye las regiones afrotropical y oriental, Australia, Europa y Oriente Medio, así como la región neotropical, Canadá, Estados Unidos y las Antillas (Harbach 2021). A pesar de esta amplia distribución, no se conoce ninguna especie para Nueva Zelanda y Nueva Caledonia (Harbach 2021). En México se han reportado once especies, una perteneciente al subgénero Pseudoficalbia y diez pertenecientes al subgénero Uranotaenia (Díaz-Nájera y Vargas 1973; Knight y Stone 1977; Darsie 1996; Bond et al. 2014; Orta-Pineda et al. 2021). En el estado de Quintana Roo se han registrado tres especies, Uranotaenia lowii Theobald, 1901, Uranotaenia sapphirina (Osten Sacken, 1868) y Uranotaenia socialis Theobald, 1901 (Díaz-Nájera y Vargas 1973; Ortega-Morales et al. 2010), sin embargo, en el registro de Ur. sapphirina realizado por Díaz-Nájera y Vargas (1973) no se señala la localidad de recolección. En esta nota se aportan nuevos registros de distribución para Ur. sapphirina basados en larvas de IV estadio recolectadas en el estado de Quintana Roo, México.

Los días 6 y 7 de abril y 4 y 5 de mayo de 2021 se realizaron colectas de larvas de culícidos como parte de las actividades de vigilancia entomológica de los Servicios Estatales de Salud

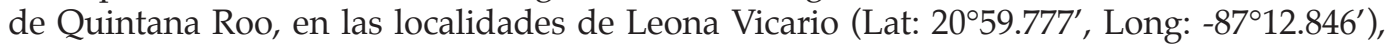
municipio de Puerto Morelos, así como Cacao (Lat: $18^{\circ} 11.415^{\prime}$, Long: $-88^{\circ} 41.478^{\prime}$ ) y Ramonal (Lat: $18^{\circ} 25.203^{\prime}$, Long: $-88^{\circ} 31.771^{\prime}$ ), municipio de Othón P. Blanco (Fig. 1). Las larvas fueron recolectadas en cuerpos de agua utilizando caladores hidro-entomológicos, posteriormente las muestras conservadas en viales con agua del lugar se trasladaron a la Unidad de Investigación Entomológica y Bioensayos de Quintana Roo (UIEB-QROO) donde se trabajó en su separación y preservación en alcohol al 70\%. La identificación taxonómica fue realizada en el Laboratorio Estatal de Salud Pública de Quintana Roo, con las claves taxonómicas de Darsie y Ward (2005) y Rivera-García et al. (2019).

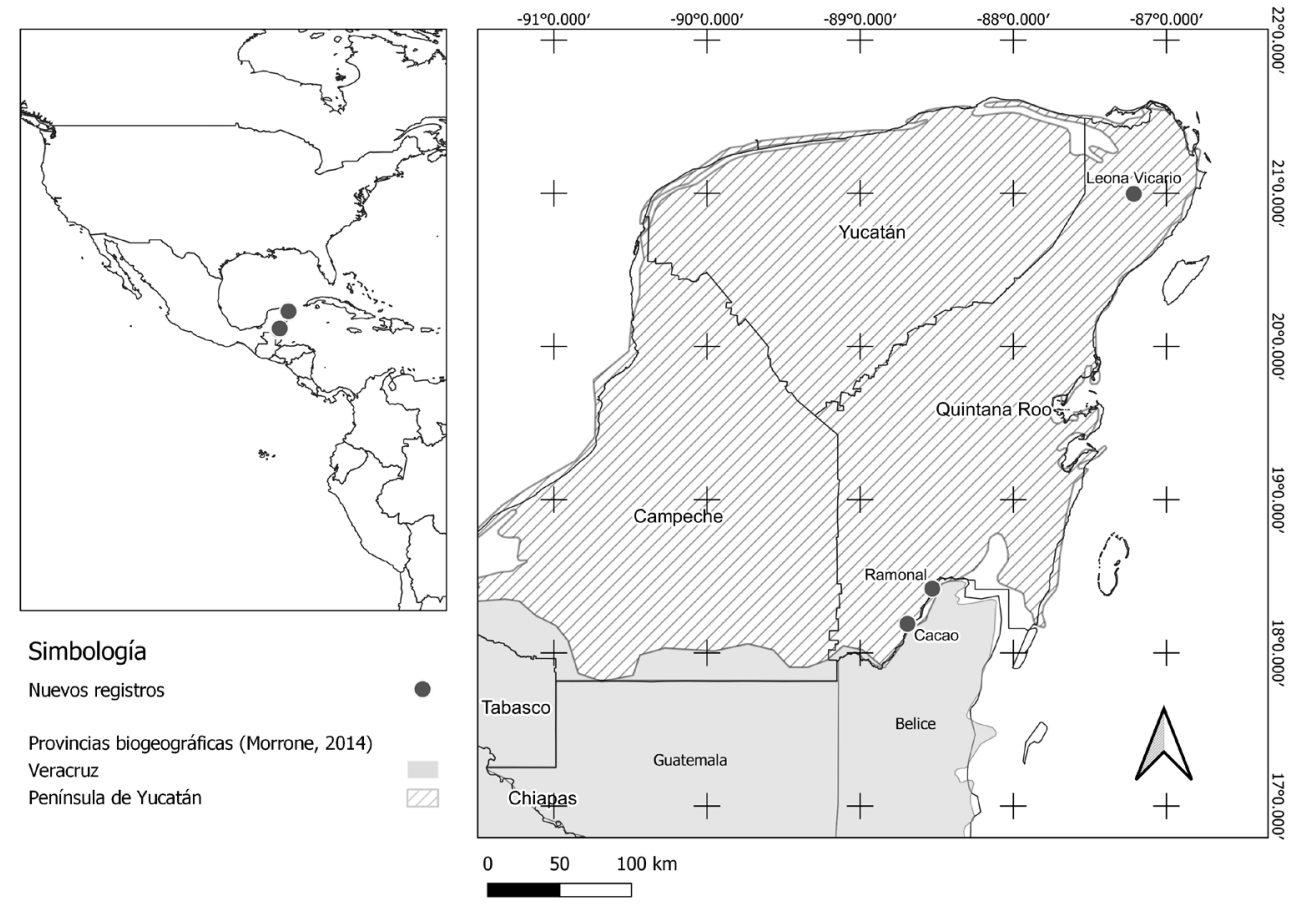

Figura 1. Sitios de recolección de Uranotaenia sapphirina en el estado de Quintana Roo, México. / Collection sites of Uranotaenia sapphirina in the state of Quintana Roo, Mexico. 
Se recolectó un total de ocho larvas de Ur. sapphirina, seis en la localidad Leona Vicario, una en Cacao y una en Ramonal. La larva de Ur. sapphirina, se diferencia de otras especies del subgénero Uranotaenia por la siguiente combinación de caracteres: Seda 3-P más corta que 0,5 la longitud de la seda 1-P; segmentos abdominales con la seda 6-I-II triple; segmento abdominal VIII con 7-10 escamas en el peine de la placa lateral; y pecten del sifón con 12-15 espinas (Fig. 2) (Darsie y Ward 2005; Rivera-García et al. 2019).

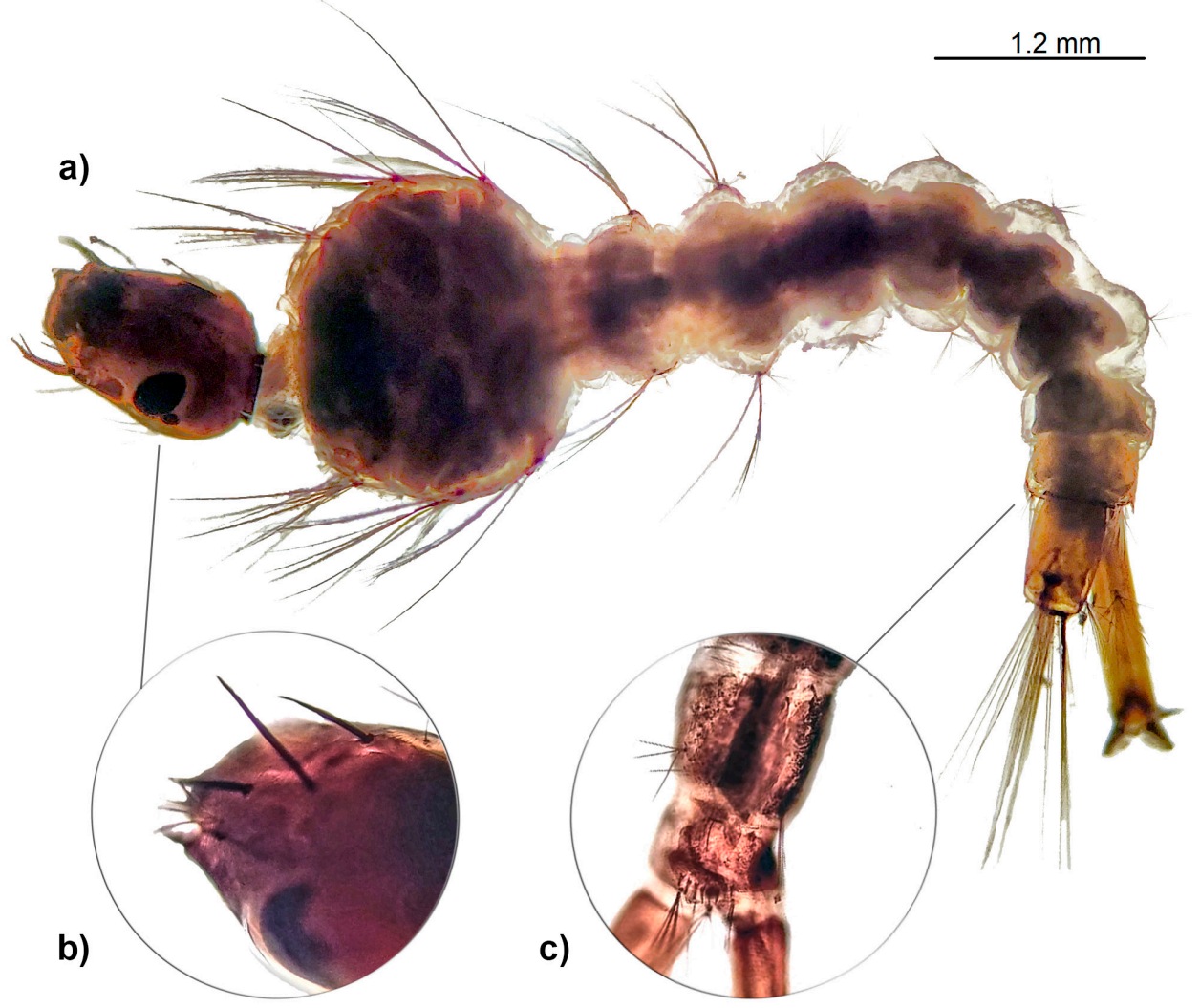

Figura 2. Larva de Uranotaenia sapphirina recolectada en Quintana Roo, México. a) Cuerpo en vista dorsal, b) Cabeza mostrando las sedas 5-6-C, y c) Segmento abdominal VIII mostrando las escamas del peine en la placa lateral. / Larva of Uranotaenia sapphirina collected in Quintana Roo, Mexico. a) Body in dorsal view, b) Head showing setae 5-6-C, and c) Abdominal segment VIII showing comb scales on lateral plate.

La distribución de Ur. sapphirina en las Américas incluye a Canadá, República Dominicana, El Salvador, Haití, México, Puerto Rico, Surinam y Estados Unidos (WRBU 2021). En el territorio mexicano ha sido recolectada en los estados de Chiapas, Colima, Guerrero, Morelos, Tabasco, Veracruz (Martini 1935), Michoacán, Ciudad de México y Sinaloa (Vargas 1956), Campeche, Jalisco, Oaxaca, Quintana Roo, Tamaulipas (Díaz-Nájera y Vargas 1973), Coahuila (Ibáñez-Bernal y Martínez-Campos 1994), Hidalgo (OrtegaMorales et al. 2018), Yucatán (Navarrete-Carballo et al. 2021) y en el estado de México (Adeniran et al. 2021). El registro previo de Ur. sapphirina en Quintana Roo data de hace 48 años (Díaz-Nájera y Vargas 1973), y en él no se mencionó la localidad de captura, no siendo recolectada nuevamente en los últimos años. En este estudio, las larvas de Ur. sapphirina fueron recolectadas junto a larvas de Anopheles (Nyssorhynchus) albimanus Wiedemann, 1820, Anopheles (Ano.) pseudopunctipennis Theobald, 1901 y Culex (Melanoconion) erraticus (Dyar y Knab, 1906). 
Anteriormente, Ortega-Morales et al. (2010) y Chan-Chable et al. (2020) realizaron actualizaciones en el listado de especies de Culicidae del Estado, sin embargo, Ur. sapphirina fue omitida dentro de las mismas debido probablemente a que no se contaba con la ubicación exacta de su presencia, por lo que en este aporte se proveen los primeros registros puntuales de Ur. sapphirina en Quintana Roo (norte y sur), incorporándola al listado de Culicidae del Estado, con la cual se alcanza un total de 87 especies.

Las especies de Uranotaenia rara vez pican a los humanos, por lo que se conoce poco sobre su importancia médica. Sin embargo, Ur. sapphirina ha sido señalada como vector potencial del virus de la encefalitis equina del Este y el virus del Nilo Occidental (Andreadis et al. 2004; Molaei et al. 2016; Reeves et al. 2018). Estudios recientes documentan que las hembras de Ur. sapphirina se alimentan de anélidos (lombrices de tierra y sanguijuelas), ampliando la gama de taxones hospedantes de Culicidae, los que a su vez pueden jugar un papel importante en la transmisión de los patógenos asociados a estos insectos (Reeves et al. 2018).

\section{Agradecimientos}

Al personal técnico de entomología de los Servicios Estatales de Salud de Quintana Roo por su valioso apoyo en la colecta del material biológico. A Emanuel Gómez-Jaramillo por su apoyo en el trabajo del laboratorio entomológico.

\section{Literatura Citada}

Adeniran, A.A., Hernández-Triana, L.M., Ortega-Morales, A.I., Garza-Hernández, J.A., de la Cruz-Ramos, J., Chan-Chable, R.J., Vázquez-Marroquín, R., Huerta-Jiménez, H., Nikolova, N.I., Fooks, A.R. y Rodríguez-Pérez, M.A. (2021) Identification of mosquitoes (Diptera: Culicidae) from Mexico State, Mexico using morphology and COI DNA barcoding. Acta Tropica, 213: 105730.

Andreadis, T.G., Anderson, J.F., Vossbrinck, C.R. y Main, A.J. (2004) Epidemiology of West Nile virus in Connecticut: a five-year analysis of mosquito data 1999-2003. Vector-Borne and Zoonotic Diseases, 4(4): 360-378.

Bond, J.G., Casas-Martínez, M., Quiroz-Martínez, H., Novelo-Gutiérrez, R., Marina, C.F., Ulloa, A. y Williams, T. (2014) Diversity of mosquitoes and the aquatic insects associated with their oviposition sites along the Pacific coast of México. Parasites and Vectors, 7(1): 1-19.

Burkett-Cadena, N.D. (2013) Mosquitoes of the southeastern United States. University of Alabama Press, Tuscaloosa, Alabama. 208 pp.

Chan-Chable, R.J., Martínez-Arce, A., Ortega-Morales, A.I. y Mis-Ávila, P.C. (2020) New records and updated checklist of mosquito species in Quintana Roo, Mexico, using DNA-Barcoding. Journal of the American Mosquito Control Association, 36(4): 264268.

Darsie Jr, R.F. (1996) A survey and bibliography of the mosquito fauna of México (Diptera: Culicidae). Journal of the American Mosquito Control Association, 12(2): 298-306.

Darsie Jr, R.F. y Ward, R.A. (2005) Identification and geographical distribution of the mosquitoes of North America, North of Mexico. University of Florida Press, Gainesville, Florida. 398 pp.

Díaz-Nájera, A. y Vargas, L. (1973) Mosquitos mexicanos. Distribución geográfica actualizada. Revista de Investigación en Salud Pública, 33: 111-125.

Harbach, R.E. (2021) Uranotaenia Lynch Arribálzaga, 1891. Mosquito Taxonomic Inventory. Disponible en: http:// mosquito-taxonomic-inventory.info/ (consultado 01 de julio 2021). 
Hoshi, T., Higa, Y. y Chaves, L.F. (2014) Uranotaenia novobscura ryukyuana (Diptera: Culicidae) population dynamics are denso-dependent and autonomous from weather fluctuations. Annals of the Entomological Society of America, 107(1): 136-142.

Ibáñez-Bernal, S. y Martínez-Campos, C. (1994) Clave para la identificación de larvas de mosquitos comunes en las áreas urbanas y suburbanas de la República Mexicana (Diptera: Culicidae). Folia Entomológica Mexicana, 92: 43-73.

Knight, K.L. y Stone, A. (1977) Catalog of the mosquitoes of the world (Diptera: Culicidae). Entomological Society of America. 611 pp.

Martini, E. (1935) Los Mosquitos de México. Departamento de Salubridad Pública. Boletines Técnicos. Serie A: Entomología Médica y Parasitología. No. 1. México, D. F. 66 pp.

Molaei, G., Thomas, M.C., Muller, T., Medlock, J., Shepard, J.J., Armstrong, P.M. y Andreadis, T.G. (2016) Dynamics of vector-host interactions in avian communities in four eastern equine encephalitis virus foci in the northeastern US. PLoS Neglected Tropical Diseases, 10(1): e0004347.

Navarrete-Carballo, J., Chan-Espinoza, D., Huerta, H., Trujillo-Peña, E., López-Platas, J., Vivas-Pérez, D., Damasco-Córdova, K., Medina-Barreiro, A., Delfín-González, H., Manrique-Saide, P. y Martin-Park, A. (2021) Diversity of Culicidae and Tabanidae (Diptera) and new record of Uranotaenia sapphirina from the archaeological site of X'cambó, Yucatan, Mexico. International Journal of Tropical Insect Science, 41(2): 1355-1363.

Orta-Pineda, G., Abella-Medrano, C.A., Suzán, G., Serrano-Villagrana, A., y OjedaFlores, R. (2021) Effects of landscape anthropization on sylvatic mosquito assemblages in a rainforest in Chiapas, Mexico. Acta Tropica, 216: 105849.

Ortega-Morales, A.I., Mis Ávila, P., Elizondo-Quiroga, A., Harbach, R.E., SillerRodríguez, Q.K. y Fernández-Salas, I. (2010) The mosquitoes of Quintana Roo State, Mexico (Diptera: Culicidae). Acta Zoológica Mexicana (nueva serie), 26(1): 33-46.

Ortega-Morales, A.I, Zavortink, T., Huerta-Jiménez, H., Ibáñez-Bernal, S. y SillerRodríguez, K. (2018) The mosquitoes (Diptera: Culicidae) of Hidalgo state, Mexico. Acta Tropica, 189: 94-103.

Reeves, L.E., Holderman, C.J., Blosser, E.M., Gillet-Kaufman, J.L., Kawahara, A.Y., Kaufman, P.E. y Burkett-Cadena, N.D. (2018) Identification of Uranotaenia sapphirina as a specialist of annelids broadens known mosquito host use patterns. Communications Biology, 1(92): 1-8. https:/ / doi.org/10.1038/s42003-018-0096-5

Rivera-García, K.D., Rísquez-Pérez, A. y Ibáñez-Bernal, S. (2019) Description of the pupa and additional characters of the fourth-instar larva, female, and male genitalia of Uranotaenia (Uranotaenia) coatzacoalcos Dyar \& Knab, with keys for the identification of Mexican species of Uranotaenia (Diptera: Culicidae). Zootaxa, 4608(2): 247-260.

Vargas, L. (1956) Especies y distribución de mosquitos mexicanos no anofelinos (Insecta Diptera). Revista del Instituto de Salubridad y Enfermedades Tropicales, 16(1): 19-36.

WRBU (Walter Reed Biosystematics Unit) (2021) Systematic Catalog of Culicidae. Disponible en: https: / / www.wrbu.si.edu (consultado 13 de julio 2021). 